\title{
AN INEQUALITY SUGGESTED BY LITTLEWOOD
}

\author{
GRAHAME BENNETT
}

ABSTRACT. It is shown that

$$
\sum_{n} a_{n}^{3} \sum_{m=1}^{n} a_{m}^{2} \sum_{k=1}^{m} a_{k} \leq \frac{3}{2} \sum_{n} a_{n}^{4}\left[\sum_{k=1}^{n} a_{k}\right]^{2}
$$

for arbitrary nonnegative numbers $a_{1}, a_{2}, \ldots$

The purpose of this note is to prove the following inequality:

$$
\sum_{n} a_{n}^{3} \sum_{m=1}^{n} a_{m}^{2} A_{m} \leq K \sum_{n} a_{n}^{4} A_{n}^{2} .
$$

Here the $a_{n}$ 's are arbitrary nonnegative numbers with partial sums $A_{n}=a_{1}+\cdots+$ $a_{n}$ and $K$ is an absolute constant.

The question as to whether any such $K$ exists was offered by Littlewood [3, p.152] as an example of an elementary inequality that is "not easy to decide." Littlewood's paper contains several problems of a similar kind; (1) is perhaps the simplest of these, and certainly the simplest to state. His inequalities all have their roots in the theory of orthogonal series, but they are pursued for their own sake in [3]. The work on orthogonal series, to which Littlewood refers, appears in an unpublished $\mathrm{Ph}$. D. dissertation of Bray [2]. Owing to the technical nature of this analysis, we must refer the reader to [3] for the origins of (1).

Our proof of (1) has no application to orthogonal series, and, in fact, no new techniques are involved. On the other hand, our search for a proof of (1) has led to several new inequalities which are of interest in their own right. These inequalities will be described elsewhere [1].

We actually prove a more general result than (1). Setting $p=2$ and $q=1$ in the theorem below, and interchanging the order of summation on the left of (3), shows that (1) holds with $K=3 / 2$. Furthermore, setting $p=1$ and $q=2$ leads to

$$
\sum_{n} a_{n} A_{n}^{2}\left[\sum_{m \geq n} a_{m}^{3 / 2}\right]^{2} \leq 4 \sum_{n} a_{n}^{2} A_{n}^{4},
$$

and this settles a second problem of Littlewood [3, p. 152].

I do not know whether the constants appearing in (1), (2), and (3) are best possible; machine calculations indicate that they are not.

Received by the editors March 14, 1986.

1980 Mathematics Subject Classification (1985 Revision). Primary 26D15.

These results, and those of [1], were presented at the Ohio Informal Analysis Seminar, November 1986. The author wishes to thank Professors Diestel, Fridy and Tonge for their hospitality. 
THEOREM. Let $p, q \geq 1$. Then

(3)

$$
\sum_{n} a_{n}^{p} A_{n}^{q}\left[\sum_{m \geq n} a_{m}^{1+p / q}\right]^{q} \leq\left[\frac{2 p-1}{p} q\right]^{q} \sum_{n}\left[a_{n}^{p} A_{n}^{q}\right]^{2} .
$$

PROOF. Our proof involves just two applications of Hölder's inequality; the only point of difficulty lies in the choice of exponents.

We may assume that only finitely many of the $a_{n}$ 's are positive, say $a_{n}=0$ whenever $n>N$. To keep the notation manageable, we set $b_{n}=a_{n} A_{n}^{q / p}$ and $c_{n}=\sum_{m \geq n} a_{m}^{1+p / q}$. Elementary estimates give

$$
A_{n}^{1+q / p} \geq b_{1}+\cdots+b_{n} \quad\left(=B_{n} \text { say }\right)
$$

and

$$
c_{n}^{r}-c_{n+1}^{r} \leq r c_{n}^{r-1} a_{n}^{1+p / q},
$$

where $r(\geq 1)$ is to be chosen later.

Letting $\theta=(2 p-1)^{-1}$ so that $0<\theta \leq 1$, the left-hand side of (3) may be rewritten as

$$
L=\sum_{n} b_{n}^{p(1-\theta)} \cdot b_{n}^{p \theta} c_{n}^{q}
$$

Applying Hölder's inequality with exponents $2 /(1-\theta)$ and $2 /(1+\theta)$, we have

$$
\begin{aligned}
L & \leq\left[\sum_{n} b_{n}^{2 p}\right]^{(1-\theta) / 2}\left[\sum_{n} b_{n}^{2 p \theta /(1+\theta)} c_{n}^{2 q /(1+\theta)}\right]^{(1+\theta) / 2} \\
& =\left[\sum_{n} b_{n}^{2 p}\right]^{(1-\theta) / 2}\left[\sum_{n} b_{n} c_{n}^{r}\right]^{(1+\theta) / 2}
\end{aligned}
$$

where we have set

$$
r=2 q /(1+\theta)=(2 p-1) q / p \geq 1 .
$$

Thus, to prove (3), it suffices to show that

$$
\sum_{n} b_{n} c_{n}^{r} \leq r^{r} \sum_{n} b_{n}^{2 p}
$$

Summing by parts, and then applying (4) and (5), we see that

$$
\begin{aligned}
\sum_{n=1}^{N} b_{n} c_{n}^{r} & =\sum_{n=1}^{N-1} B_{n}\left[c_{n}^{r}-c_{n+1}^{r}\right]+B_{N} c_{N}^{r} \\
& \leq r \sum_{n=1}^{N} a_{n}^{1+p / q} A_{n}^{1+q / p} c_{n}^{r-1} \\
& =r \sum_{n=1}^{N} b_{n}^{1 / r^{*}} c_{n}^{r-1} \cdot b_{n}^{2 p / r}
\end{aligned}
$$


where $r^{*}=r /(r-1)$ is the conjugate of $r$ (see (6)). Applying Hölder's inequality once more gives

$$
\sum_{n} b_{n} c_{n}^{r} \leq r\left[\sum_{n} b_{n} c_{n}^{r}\right]^{1 / r^{*}}\left[\sum_{n} b_{n}^{2 p}\right]^{1 / r}
$$

which is equivalent to (7).

\section{REFERENCES}

1. G. Bennett, Some elementary inequalities, Quart. J. Math. (to appear).

2. J. Bray, Ph.D. thesis, Cambridge University.

3. J. E. Littlewood, Some new inequalities and unsolved problems, Inequalities (Ed., O. Shisha), Academic Press, New York, 1967, pp. 151-162.

Department of Mathematics, SWAin Hall-East, Indiana UNiVERSity, BloOMINGTON, INDIANA 47405 